\title{
Condominios und fraccionamientos cerrados in Mexiko-Stadt - Sozialräumliche Segregation am Beispiel abgesperrter Wohnviertel
}

\section{Sigrun Kanitscheider, Neustift}

\section{Einleitung}

Das Phänomen Segregation gibt es genauso lange wie städtische Siedlungen existieren, soziale, wirtschaftliche, vor allem auch ethnische und religiöse Unterschiede können sich in der räumlichen Verteilung der städtischen Bewohner widerspiegeln. Armen- und Reichenviertel kennzeichnen Städte in allen Kontinenten. In vielen Fällen ist die sozialräumliche Segregation mit der Bildung von Ghettos oder Slums verbunden, in denen soziale oder ethnische Minderheiten unfreiwillig vom Rest der städtischen Bevölkerung getrennt sind.

In den Städten Lateinamerikas findet man Segregation jedoch in erster Linie als Ergebnis der Unterschiede zwischen Arm und Reich. Die in den letzten Jahrzehnten immer drastischer gewordenen sozialen Gegensätze, charakterisiert vor allem durch ein prozentuales Anwachsen der Marginalbevölkerung, haben zu einer weiteren Fragmentierung des städtischen Raumes geführt (CABRALES \& CANOSA 2001: 223).

Für die wohlhabende Minderheit führen die Angst vor Kriminalität und der Wunsch nach Exklusivität in Mexiko zu dem Bedürfnis, sich in den von Sicherheit und Ruhe gekennzeichneten abgeschlossenen Wohnvierteln niederzulassen. Dies zeigt sowohl die Untersuchung von CaBrales \& CANOSA (2001: 224) als auch die folgende Arbeit. Abhängig von den Anforderungen der Bewohner zeigen sich charakteristische Unterschiede in Lage, Größe und Infrastruktur, die dem Phänomen unterschiedliche Erscheinungsformen geben. Mehr noch als in anderen lateinamerikanischen Städten könnte in Mexiko aufgrund der Nähe und der starken wirtschaftlichen und sozialen Verflechtung bei der Entstehung dieser Viertel eine Beeinflussung durch die USA vermutet werden. Um diese Hypothese zu stützen, wäre eine Untersuchung der Verflechtungen zwischen den Immobilienmärkten Mexikos und der Vereinigten Staaten nötig.

Während diese von der Öffentlichkeit mit Mauern, Zäunen und Wachpersonal abgeschirmten geschlossenen Siedlungen wissenschaftlich noch kaum untersucht worden sind, bilden sie in der nordamerikanischen Forschung längst ein zentrales Erkenntnisinteresse. Dort werden solche Wohnviertel gated communities genannt; in Lateinamerika, wo sich seit den 70er Jahren diese abgesperrten Wohnsiedlungen entwickeln, gibt es keinen einheitlichen Begriff für das Phänomen. Während in Chile und Brasilien die Bezeichnung condominio verwendet wird, spricht man in Argentinien von barrios privados, in Ecuador von conjuntos oder urbanizaciones cerradas. In Mexiko sind als Bezeichnung condominio cerrado, fraccionamiento cerrado und coto cerrado in Gebrauch, teilweise allerdings mit unterschiedlicher Bedeutung. Im folgenden wird der Terminus barrios cerrados verwendet, um die sich in ihrem Rechtsstatus voneinander unterscheidenden Formen der geschlossenen Wohnsiedlungen zusammenzufassen.

Obwohl es sich bei den barrios cerrados durchaus um in bezug auf Stadtstruktur und -entwicklung relevante Phänomene handelt, werden sie bisher in der öffentlichen Diskussion kaum wahrgenommen. In Mexiko beschränkt sich der Diskurs auf die akademische Ebene. Wenngleich die ersten Beispiele für barrios cerrados in Lateinamerika schon in den 1970er Jahren entstehen, erschienen erst in den letzten Jahren die ersten Publikationen darüber. So wurden in Santiago de Chile (Borsdorf 1998, 2000; Meyer \& BäHr 2001), Rio de Janeiro (PöHLER 1999; CoY \& PöHLER 2001), Buenos Aires (SuÁrez 1997; JanoschKa 2000), Mexiko-Stadt (SAFA 1999) und Guadalajara (CABRALES \& CANOSA 2001) Untersuchungen zu den barrios cerrados durchgeführt, die sich jedoch meist auf Fallstudien beschränken.

Aufgrund der fehlenden Erfassung der geschlossenen Wohnsiedlungen von öffentlicher Seite gibt es auch in Mexiko keine offiziellen Statistiken, die die Bedeutung des Phänomens für die Stadt abschätzen ließen. In diesem Artikel wird nun versucht, aufgrund eigener Untersuchungen in Mexiko-Stadt auf folgende Fragen einzugehen: Welche sozialen Schichten sind betroffen, und was sind die Motive für einen Umzug in barrios cerrados? Gibt es bevorzugte Gebiete für die Ansiedlung von barrios cerrados, entstehen in Folge davon Typen, abhängig von der Lage im Raum, und lassen sich durch die Absperrung kleinräumigere Segregationsmuster feststellen? Und schließlich, wie sieht die zeitliche Perspektive aus?

\section{Methodik der Erhebungen}

Für die Erhebung der Daten, die dieser Arbeit zugrunde liegen, wurden sowohl qualitative wie auch 
quantitative Forschungsmethoden verwendet. Obwohl die Erkenntnisse aus bisherigen Studien in nordamerikanischen und lateinamerikanischen Städten durchaus ein an Theorien und Arbeitshypothesen orientiertes Vorgehen erlaubt hätten, folgt die vorliegende Untersuchung einer induktiv-explorativen Logik, um unvermutete Zusammenhänge aufzudekken. Vielfach war die Feldarbeit dabei von der praktischen Überlegung bestimmt, welche Informationen mit den zur Verfügung stehenden Mitteln eingeholt werden können.

Bei der Erfassung der Information kamen verschiedene Methoden zum Einsatz: leitfadenorientierte Interviews mit Experten aus Universität, narrative Gespräche mit Spezialisten aus Politik und Immobiliengeschäft und Befragungen von Bewohnern mittels eines standardisierten Fragebogens. Bei der Auswahl der untersuchten barrios cerrados wurde auf eine größtmögliche Varietät sowohl in räumlicher als auch in sozialer Hinsicht geachtet, um dem breiten Spektrum gerecht zu werden.

\section{Barrios cerrados in der Stadtstruktur von Mexiko}

In den wichtigsten lateinamerikanischen Großstädten stellt diese relativ junge Wohnform bereits ein für europäische Augen auffallendes Charakteristikum der Stadtstruktur dar. Grund dafür ist, wie eine Arbeit zumindest für Mexiko nachweist (CABRales \& CANOSA 2001: 234), eine enorme Ungleichheit der Einkommensverhältnisse, die das Bedürfnis nach privater Sicherheit und Exklusivität fördert, denn Straftaten sind

«Folgen unterschiedlicher sozialer Faktoren ... Arbeitslosigkeit, Armut, Ungleichheit, fehlende Bildung, ungeordnetes Wachstum der Stadt werden als Ursache für den Anstieg der Kriminalität genannt” (Beltrán \& Piccato 2000: 8; Übersetzung d. Verf.).

Auch wenn es bislang noch keine eindeutigen Untersuchungen diesbezüglich gibt, deutet vieles darauf hin, dass der starke Anstieg der Kriminalität seit Mitte der 1990er Jahre das Entstehen von abgeschlossenen Wohnsiedlungen besonders gefördert hat.

In Mexiko entwickelten sich die ersten Vorformen der heute sich so stark ausbreitenden barrios cerrados Ende der 1960er Jahre in der äußeren Umgebung der Städte als sogenannte Country Clubs. Mit großzügig gebauten Einfamilienhäusern und einem üppigen Freizeit- und Dienstleistungsangebot ausgestattet, wurden diese von der Oberschicht zunächst in erster Linie für Wochenenden genutzt, mit der Verbesserung der Verkehrsinfrastruktur und dem Näherrücken der Stadtgrenze steigt später der Nutzungsgrad (CABRALES \& CANOSA 2001:230).
In den 1970er Jahren, als die Oberschicht aus dem inneren Stadtbereich an die Peripherie zu ziehen beginnt, bilden sich am Stadtrand durch Initiative einzelner Immobilienfirmen neue «Nobelviertel», die später, oft erst in den 1990er Jahren, mit entsprechenden Sicherheitsvorkehrungen versehen zu barrios cerrados werden. $\mathrm{Zu}$ einer städtebaulich relevanten Erscheinung werden diese in der mexikanischen Hauptstadt erst Ende der 1980er und in den 1990er Jahren, als nicht mehr nur die Oberschicht an dieser Wohnform interessiert ist.

Meyer \& BäHr (2001: 294) definieren den Begriff barrio cerrado als eine

«erkennbare Einheit im Siedlungsraum, die durch Zäune oder Mauern sowie Sicherheitseinrichtungen gegen unbefugtes Betreten geschützt ist, also in der Regel rund um die Uhr bewacht ist, über eine mehr oder weniger große Zahl gemeinsamer Einrichtungen verfügt und die eine gewisse Mindestgröße hat, d.h. mehrere Haushalte aufweist.»

In der Literatur wurden bisher vor allem zwei städtebauliche Grundformen von barrios cerrados klassifiziert (MEYER \& BäHR 2001: 294): Die aus Einfamilienhäusern zusammengesetzten Wohnsiedlungen (condominios horizontales) mit einer alle Privatgrundstücke umschließenden Mauer und die in mehr als zwei Hochhäusern angeordneten Wohnungen (condominios verticales) mit Umzäunung des gemeinschaftlichen Grundstückes und Sicherheitskontrolle. In der Stadt Mexiko bestehen etwa zwei Fünftel der in den 1990er Jahren errichteten barrios cerrados aus Einfamilienhäusern, der etwas größere Teil also aus Wohnungen in Mehrfamilienhäusern (SOFTEC 1989-2001).

\subsection{Der rechtliche Aspekt}

Abgesehen von der morphologischen Differenzierung sind auch die juristischen Unterschiede nach mexikanischem Recht bedeutend (vgl. Foto 1): Condominios werden zur Gänze auf privatem Grund errichtet; d.h. neben den einzelnen Wohneinheiten werden auch die entsprechenden Anteile der notwendigen Infrastruktur verkauft. Deshalb führt die Absperrung der (privaten) Straße zu keiner Rechtsverletzung. Im Gegensatz dazu gehören in fraccionamientos die Straßen, Parks etc. zum öffentlichen Raum. Dort steht eine Zugangsbeschränkung daher immer in Konflikt mit dem Gesetz.

Obwohl die Rechtsprechung für das Schließen einer Straße eine Strafe in dreißigfacher Höhe des Mindestlohnes vorsieht (Gobierno del Distrito FEdERAL 1999), lassen sich die Anwohner davon nicht abschrekken. Daher gehört das Aufstellen und Wiederentfernenlassen von Schutzgittern und Wachhäuschen in vielen Fällen zum Alltag. 


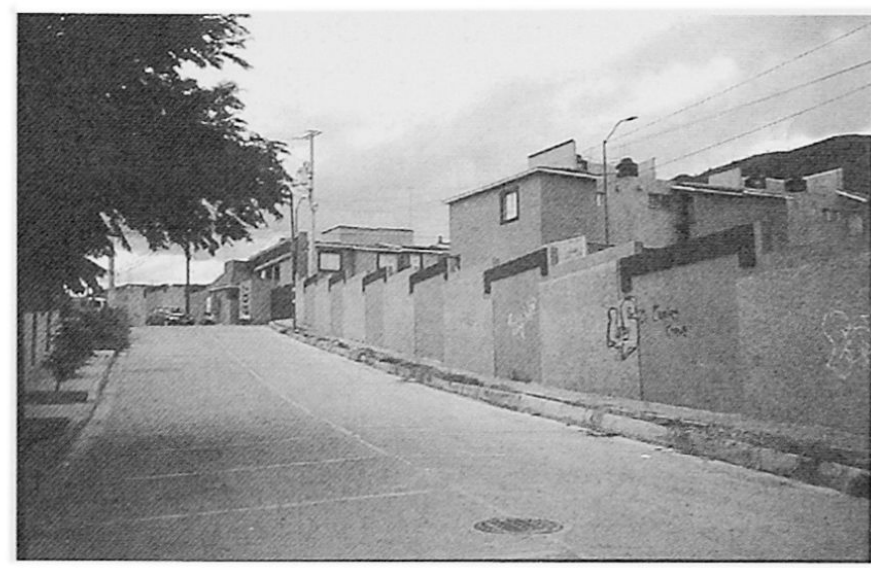

Foto 1: Condominio in Mexiko-Stadt: Mauern trennen den öffentlichen vom privaten Raum.

"Condominio" in Mexico-City: The wall separates public and private space

"Condominio" à Mexico-Cité: Des murs séparent espaces publique et privé.

Foto: A. BORSDORF

\subsection{Der soziale Aspekt}

Während die ersten geschlossenen Wohnanlagen ausschließlich für die Oberschicht gebaut wurden, ist inzwischen das Interesse der Bevölkerung aus mittleren Einkommensklassen erheblich gestiegen. Mittlerweile sind die barrios cerrados zu einer große Bevölkerungsteile betreffenden Erscheinung geworden:

«Der Kampf für eine bessere Lebensqualität ist nicht allein eine Bewegung der Reichen, die einfache Bevölkerung und die durch die Krise der letzten zwei Jahrzehnte verarmte Mittelschicht schließen sich zusammen, um Projekte für die Verbesserung ihrer Lebensbedingungen umzusetzen» (SAFA 1999: 2; Übersetzung d. Verf.).

Tatsächlich finden sich auch unter den mit öffentlichen Geldern subventionierten Wohnanlagen immer öfter auf private Initiative hin angebrachte Sicherheitseinrichtungen (Mauern, Zäune, gesicherte Tore).

Die Frage, welche sozialen Schichten sich tatsächlich in barrios cerrados niederlassen, führt letztendlich zu einem Definitionsproblem, denn während sich Oberschichtsiedlungen durch ihre Infrastruktur und massiven Sicherheitsvorkehrungen recht deutlich von anderen Nobelvierteln absetzen, unterscheidet die subventionierten Wohnanlagen oft lediglich das selbsterrichtete Gitter am Eingang von den nicht abgesperrten Straßenzügen. Die Tatsache, dass in Mexiko-Stadt etwa $40 \%$ aller Wohnhäuser durch autoconstrucción entstehen (SOFTEC 1989-2001), also ohne jegliche Beteiligung von Architekten oder Baufirmen von den Bauherren in Eigenarbeit erstellt werden, legt die Vermutung nahe, dass nicht die Unterschicht, sondern erst die untere Mittelschicht in den staatlich unterstützten und professionell gebauten Wohnsiedlungen in relevantem Ausmaß als Bewohner von barrios cerrados zu finden ist. Die Untersuchung des Immobilienangebotes im Zeitraum von 1989-2001 zeigt, dass mehr als die Hälfte aller in condominios zum Verkauf feilgebotenen Wohneinheiten aufgrund des Kaufpreises für die Unterschicht bzw. die untere Mittelschicht bestimmt waren (Abb.1).

Wenn es nun offensichtlich für eine nicht zu übersehende Schicht der Bevölkerung attraktiv ist, sich in abgesperrten Wohnsiedlungen niederzulassen, stellt sich die Frage, was das Wohnen dort so anziehend macht. Eigene Umfragen in vier ausgewählten barrios cerrados haben durchaus unterschiedliche Motive ergeben (Abb.2): Für die Oberschicht und gehobene Oberschicht spielt besonders der Sicherheitsaspekt und das Argument, einen guten Wohnort für die Kinder zu finden, eine große Rolle, zwei inhaltlich recht nahe beieinander liegende Gründe. An dritter Stelle wird dann das Wohnen in einer landschaftlich attraktiven Umgebung genannt. In der Mittelschicht hingegen wird als wichtigster Beweggrund für den Umzug genannt, ein eigenes Haus zu besitzen, am zweithäufigsten kommt das Argument, einen guten Ort für die Kinder zu finden. Während die Oberschicht meist schon vor dem Umzug in das barrio cerrado Wohneigentum hatte, ist für viele Familien der Mittelschicht dieser Umzug mit dem Verlassen des Elternhauses und dem Erwerb eines eigenen Hauses verbunden.

\subsection{Der zeitliche Aspekt}

Wie bereits eingangs geschildert entstanden in MexikoStadt in den 1970er Jahren die ersten barrios cerrados, von einem verbreitet auftretenden Phänomen kann man aber erst in der letzten Dekade sprechen.

Aufgrund der fehlenden Erfassung des Phänomens von öffentlicher Seite ist die Abschätzung der absoluten Zahl und deren zeitlicher Entwicklung in der Metropolitanregion Mexiko schwierig. Die Metropolitanregion Mexiko (Zona Metropolitana de la Ciudad de México, ZMCM) umfasst im Jahr 1995 den Distrito Federal und 37 angrenzende municipios des Bundesstaates Mexiko (Consejo Nacional de Población 2000: 9). Für den Zeitraum von 1989-2001 ergibt die Auswertung der von SoFTEC S.C. zur Verfügung gestellten Datenbank eine Zahl von knapp 750 neuerrichteten condominios cerrados mit etwa 47.000 Wohneinheiten in der Metropolitanregion Mexiko (SoFTEC S.C. 1989-2001). Nicht erfasst wurden dabei allerdings nachträglich mit Sicherheitseinrichtungen ausgestattete condominios und im öffentlichen Raum abgesperrte Wohnviertel (fraccionamientos cerrados).

Anders als in der chilenischen Hauptstadt, in der sich seit Ende der 1970er Jahre die Tendenz zu 


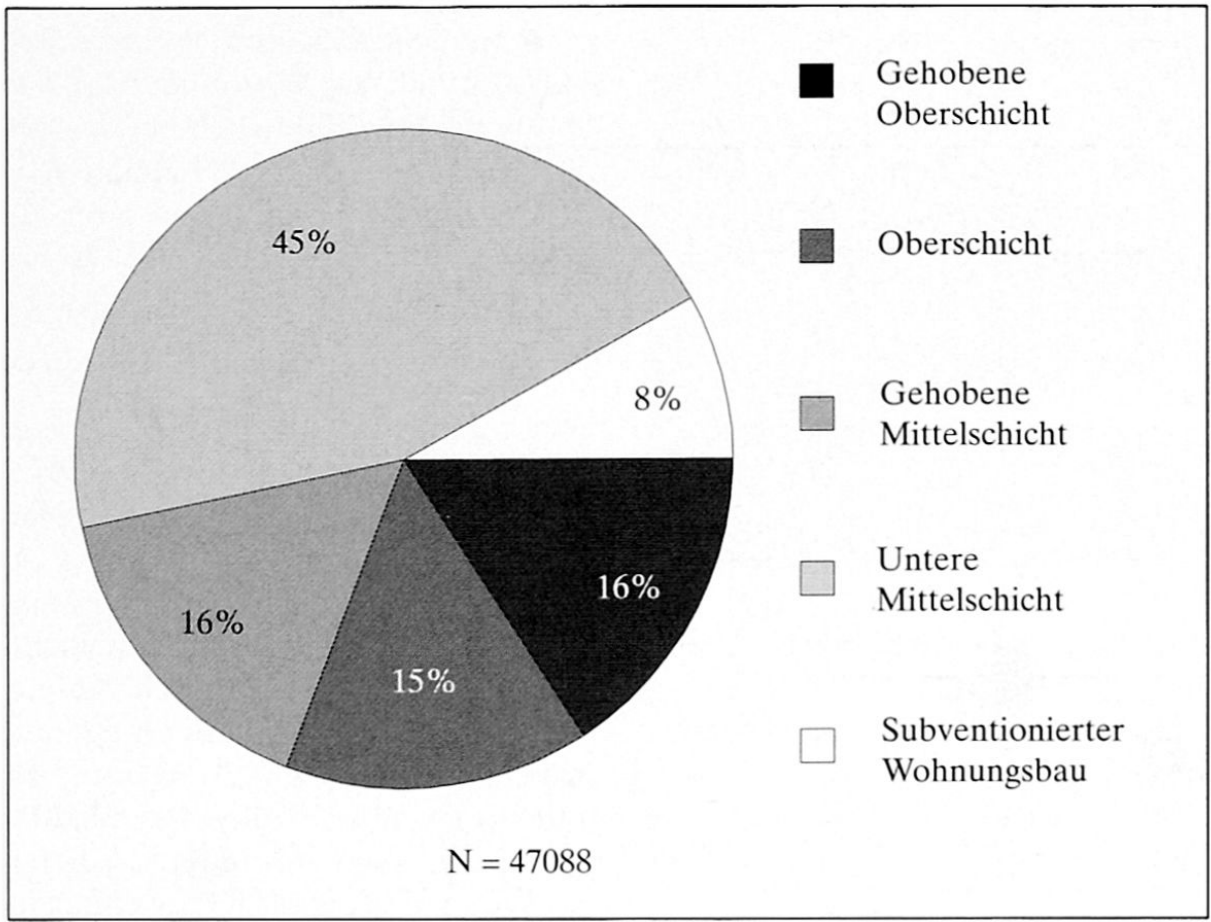

Abb. 1: Verteilung der Wohneinheiten nach Typ der condominios cerrados. Klassifizierung der Typen nach Kaufpreis.

Relative distribution of accommodation units in "condominios cerrados» in terms of purchasing price.

Distribution des unités d'habitation selon le type des "condominios cerrados». Classification des types selon le prix d'achat.

Quelle: eigene Bearbeitung nach SoFTEC S.C., Dinámica del mercado inmobiliario habitacional 1989-2001

Einfamilienhäusern als Alternative zu den Appartementtürmen feststellen lässt (BORSDORF 2000: 29), zeigt sich in der Stadt Mexiko in den genannten zwölf Jahren ein deutlicher Trend hin zu Wohnungen in Hochhäusern:Während zu Beginn der 1990er Jahre das Verhältnis zwischen neuerrichteten Wohnungen und Einfamilienhäusern relativ ausgeglichen war, nimmt der Anteil der condominios horizontales in der zweiten Hälfte der Dekade drastisch zu. Unter der Annahme, dass das Immobilienangebot in etwa die Nachfrage widerspiegelt, lässt sich daraus schließen, dass es einen deutlichen Trend zur Bevorzugung von Appartements gegenüber Einfamilienhäusern gibt. Diese Tendenz zeigt sich in den Daten über die condominios cerrados in allen sozialen Schichten gleichermaßen.

In Verbindung mit der steigenden Präferenz für Appartements scheint auch der Trend zu zentraler Wohnlage zu stehen. Wie aus Abb. 3 ersichtlich, vergrößerte sich der Anteil der im Zentrum gebauten Wohneinheiten von 1993 bis 2001 kontinuierlich, von zunächst $0 \%$ (1993) auf 30\% (2001). Diese Zunahme erfolgt nicht auf Kosten einer einzelnen anderen Zone, der Rückgang verteilt sich zeitlich ungleichmäßig auf die drei insgesamt am stärksten vertretenen Zonen Westen, Südwesten und Süd-Zentrum. Der in Zentrumsnähe knappe und relativ teure Baugrund wurde in den vergangenen Jahren vermehrt für den Bau von condominios verticales für die obere Mittelschicht und Oberschicht genutzt.

Für die Erklärung dieser relativ neuen Erscheinung der Revalorisierung zentrumsnaher Grundstücke gibt es mehrere Ansätze: Zum einen hat das starke Erdbeben von 1986 im Stadtzentrum für zahlreiche Baulücken gesorgt, die auch infolge der Wirtschaftskrise von 1994/95 noch bei weitem nicht geschlossen wurden. Viele dieser Flächen werden noch heute als Parkplätze vermietet oder wurden mittlerweile in Parks umgewandelt. Mit den sich verbessernden wirtschaftlichen Möglichkeiten der letzten Jahre, insbesondere der Erweiterung der Kreditmöglichkeiten für Immobilien durch die Banken (SOFTEC 2001: 1), wurden diese Baulücken teilweise durch Appartementhäuser geschlossen.

Zum anderen gibt es in der Stadtpolitik des Distrito Federal bereits seit einiger Zeit das Bestreben einer 




Abb. 2: Motive für das Wohnen in umzäunten Wohnformen (barrios cerrados). Erster Grund für den Umzug. Primary reason for moving to a "barrio cerrado» according to social class.

Les enclos d'habitation ou "quartiers fermés» («barrios cerrados»). Première raison du déménagement et les motivations résidentielles.

Quelle: Haushaltsbefragung, November 2001

Revitalisierung des Zentrums. Das Wachstum der Stadt soll zurück in das Stadtzentrum, insbesondere in die Delegaciones Benito Juárez, Cuauhtémoc, Miguel Hidalgo und Venustiano Carranza gelenkt werden. Damit werden diese Viertel mit einer niedrigen Bevölkerungsdichte, aber einer bereits vorhandenen Infrastruktur, "wiederverwendet», während in den längst überforderten Randzonen der Stadt die Errichtung der notwendigen Infrastruktur meist schwierig und teuer ist (Gobierno del Distrito Federal 2001: 4).

\subsection{Der räumliche Aspekt}

Unterschiedliche naturräumliche Gegebenheiten wie die von Westen in Richtung des Stadtzentrums abfallende Hanglange und die damit verbundene Abnahme der Wasserqualität der Flüsse, die Versumpfung im Gebiet des ehemals großflächigen Texcoco-Sees und die vorherrschende Windrichtung charakterisieren die natürliche Lebensqualität in der Metropole Mexiko. In
Folge davon führten die Wohnstandortpräferenzen vor allem der einkommensstärkeren Gruppen der Bürger zu einer sozialräumlichen Entmischung. Die einfache Bevölkerung siedelte in erster Linie im östlichen Teil der Stadt, während die Oberschicht sich im Westen, insbesondere im Südwesten, niederließ. Die Daten der Volkszählung von 1990 belegen diese Bevorzugung westlicher Stadtgebiete (CONSEJo Nacional de POBLACión 2000: 52). Diese räumliche Trennung von Einwohnern höherer und niedrigerer Einkommensklassen ist ein Charakteristikum der sozialen Segregation.

Bei dem Beispiel der barrios cerrados kann die physische Distanz durch starke Sicherheitseinrichtungen ersetzt werden, die ein Untersichbleiben der verschiedenen Gruppen gewährleisten. Eine Verbreitungskarte der zwischen 1989 und 2001 in der Metropolitanregion Mexiko neu errichteten Einfamilienhäuser und Wohnungen in condominios mit Sicherheitseinrichtungen 


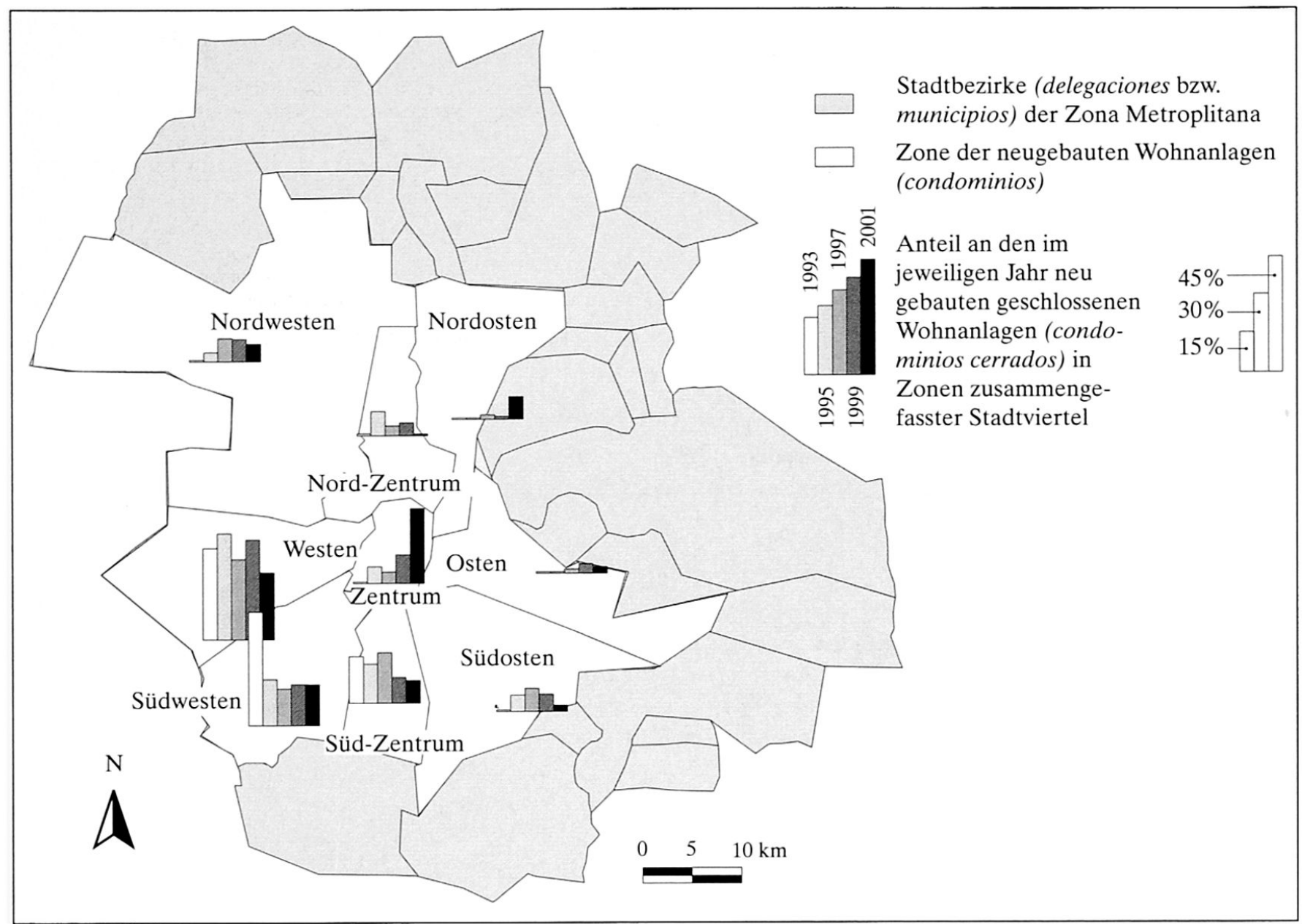

Abb. 3: Condominio cerrado-Neubauten in der Metropolitanregion Mexiko. Anteilmässige Verschiebung in verschiedenen Stadtzonen von 1993 bis 2001.

Relation of "condominios cerrados» established within the metropolitan area of Mexico 1993-2001, reflecting regional shifts.

"Condominios cerrados» nouvellement construits dans la région métropolitaine de Mexico. Evolution différenciée des proportions dans différentes zones urbaines (1993-2001).

Kartengrundlage: Instituto Nacional de Estadística, Geografía e Informatica 1992 und Softec S.C. 1989-2001; Kartographie: S. KANITSCHEIDER

(Abb. 4) zeigt, dass die angesprochene Polarisierung der Stadt ziemlich konsequent beibehalten wurde, im traditionell «reichen»Westen und Südwesten dominieren die Wohnanlagen für einkommensstärkere Teile der Bevölkerung, während im übrigen Teil der Stadt die Mittel- und die Unterschicht beinahe unter sich bleiben. Deutlich wird in dieser Karte auch, dass die Stadt Mexiko mit Ausnahme der äußersten Randzone relativ gleichmäßig von dem Phänomen der condominios cerrados betroffen ist, es gibt also keine ausgeprägte Präferenz für eine bestimmte Zone, die nicht durch die sozialen Unterschiede bedingt ist.

Betrachtet man die Verteilung dieser condominios in Kombination mit den Einkommensverhältnissen und in einem größeren Maßstab, nämlich auf Ebene der statistischen Grundeinheit AGEB (area geoestadística básica), so lassen sich jedoch kleinräumig Unterschiede feststellen. Die in den Volkszählungen seit 1990 eingeführte räumliche Untersuchungseinheit AGEB ist über Bevölkerungszahlen definiert und umfasst jeweils etwa einige tausend Einwohner. In der Delegación Álvaro Obregón, in der zwischen 1989 und 2001 absolut gesehen die meisten condominios cerrados errichtet wurden, werden die Prozentzahl der Geringverdienenden (Abb. 5) und die der Höherverdienenden einander gegenübergestellt (Abb. 6), jeweils mit der Information über Lage und Typ der in den vergangenen zwölf Jahren neuerrichteten condominios cerrados. Dabei kristallisieren sich zwei verschiedene Typen 


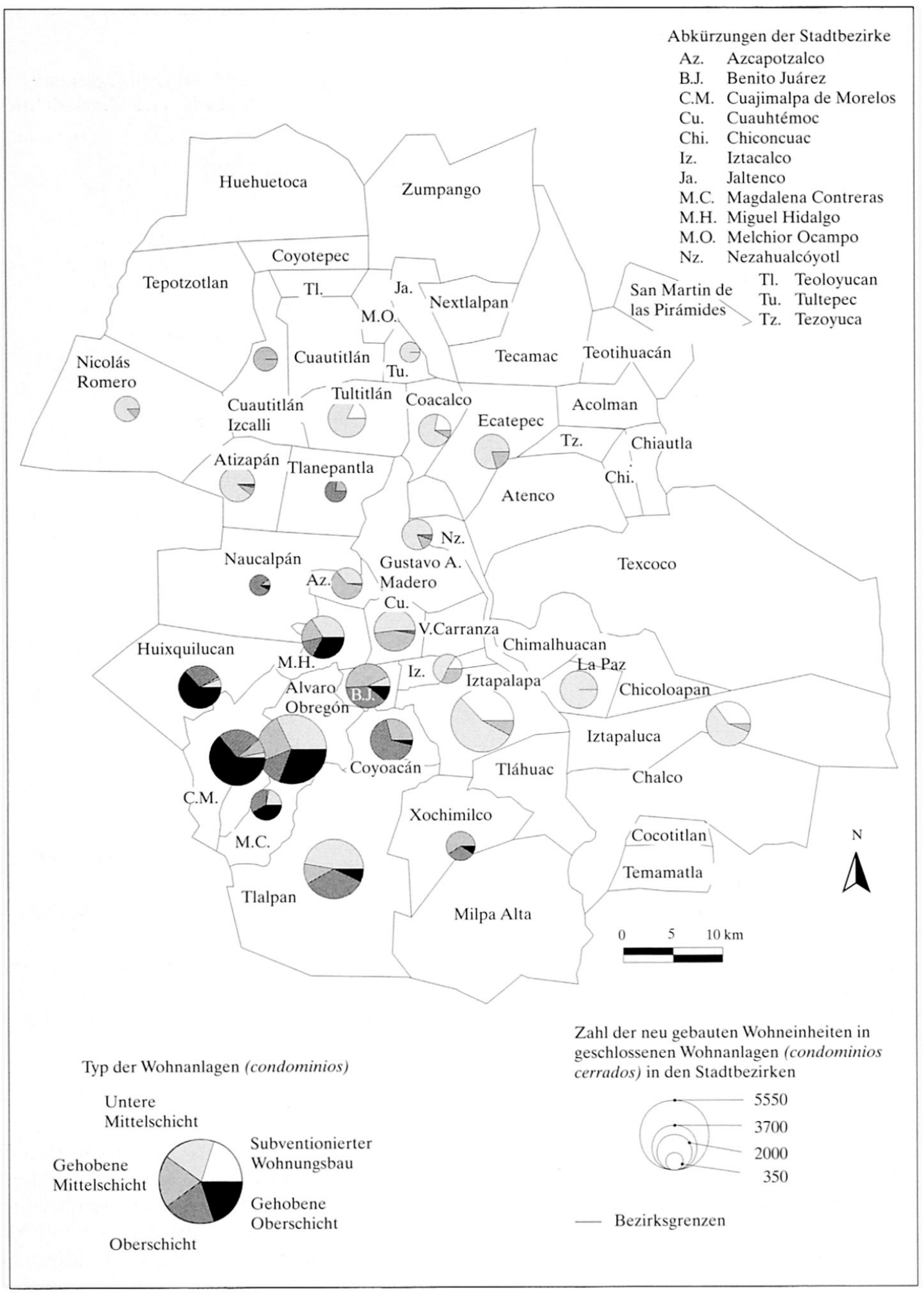

Abb. 4: Typ der von 1989-2001 in der Metropolitanregion Mexiko neu errichteten Wohneinheiten in condominios mit Sicherheitseinrichtungen.

Relative distribution of residential units in secured "condominios» built in the metropolitan area of Mexico 1989-2001, according to income groups.

Type d'unités d'habitation nouvellement construits en "condominios» dans la région métropolitaine de Mexico (1989-2001), comportant des équipements de sécurité.

Kartengrundlage: Instituto Nacional de Estadística, Geografía e Informatica 1992 und Softec S.C. 1989-2001; Kartographie: S. KANITSCHEIDER 


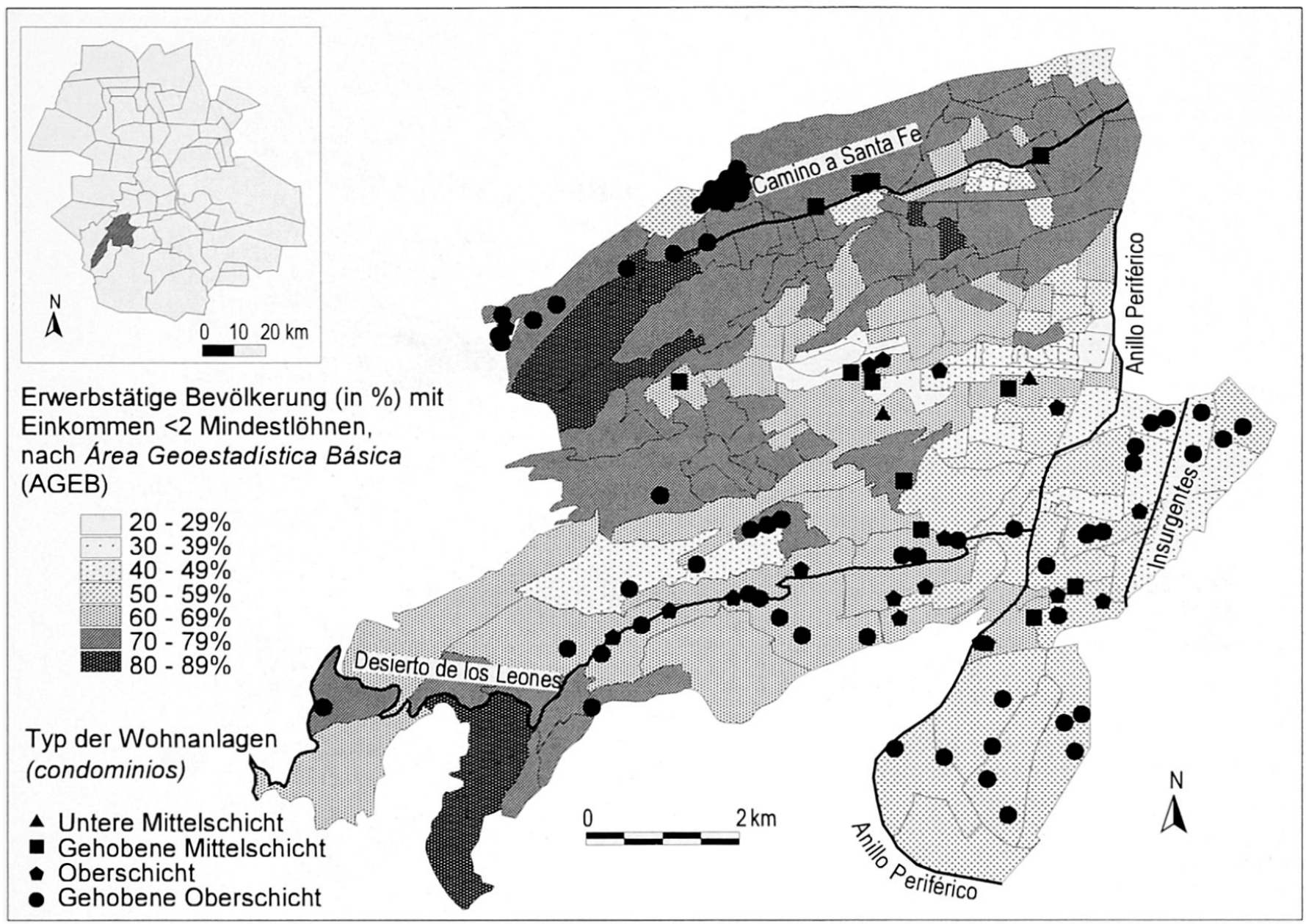

Abb. 5: Anteil der Geringverdienenden in der delegación Álvaro Obregón und Verteilung der von 1989-2001 neu gebauten condominios mit Sicherheitseinrichtungen.

Relative distribution of low income residents and distribution of secured "condominios" built 1989-2001 in the "delegación» Álvaro Obregón.

Pourcentage des gagne-petits dans la "delegación" Álvaro Obregón et répartition des "condominios» nouvellement construits, dotés d'équipements de sécurité (1989-2001).

Kartengrundlage: Instituto Nacional de Estadística, Geografía E Informatica 1992 und Softec S.C. 1989-2001; Kartographie: S. KANITSCHEIDER

von AGEBs heraus: Jene, die in den beiden Darstellungen komplementär sehr helle bzw. dunkle Grauwerte aufweisen, kennzeichnen sich durch eine große Homogenität in Bezug auf die Einkommen. Diese relativ einheitlich höheren bzw. niedrigeren Einkommen sind Anzeichen einer starken sozialen Segregation. Die AGEBs aber, die in beiden Darstellungen mittlere Werte annehmen, also weder einen ausgeprägt hohen Prozentsatz an Geringverdienenden noch an Höherverdienenden aufweisen, zeichnen sich durch Heterogenität der Einkommensverhältnisse und damit auch der sozialen Schichten aus. Die große Zahl der condominios cerrados der Oberschicht und gehobenen Oberschicht befindet sich nun nicht in den erstgenannten «reinen Reichenvierteln», sondern in den «gemischten» AGEBs mit höher- und geringer verdienender Bevölkerung. In einigen Vierteln erzeugen dabei die in diesen Oberschichthaushalten zahlreich wohnenden Dienstmädchen, Gärtner und Chauffeure in den statistischen Daten einen Eindruck von sozialer Heterogenität, der sich im Erscheinungsbild des Wohnviertels nicht widerspiegelt.

Es wird deutlich, dass die Immobilienfirmen der Kundennachfrage entsprechend bei der Errichtung neuer geschlossener Wohnanlagen sich sehr wohl an die großräumige West-Ost-Trennung halten; für diese sorgen im Falle der Mittelschicht-condominios vor allem die gehobenen Grundstückspreise im Westen und Südwesten. Untersucht man die Stadtbe- 


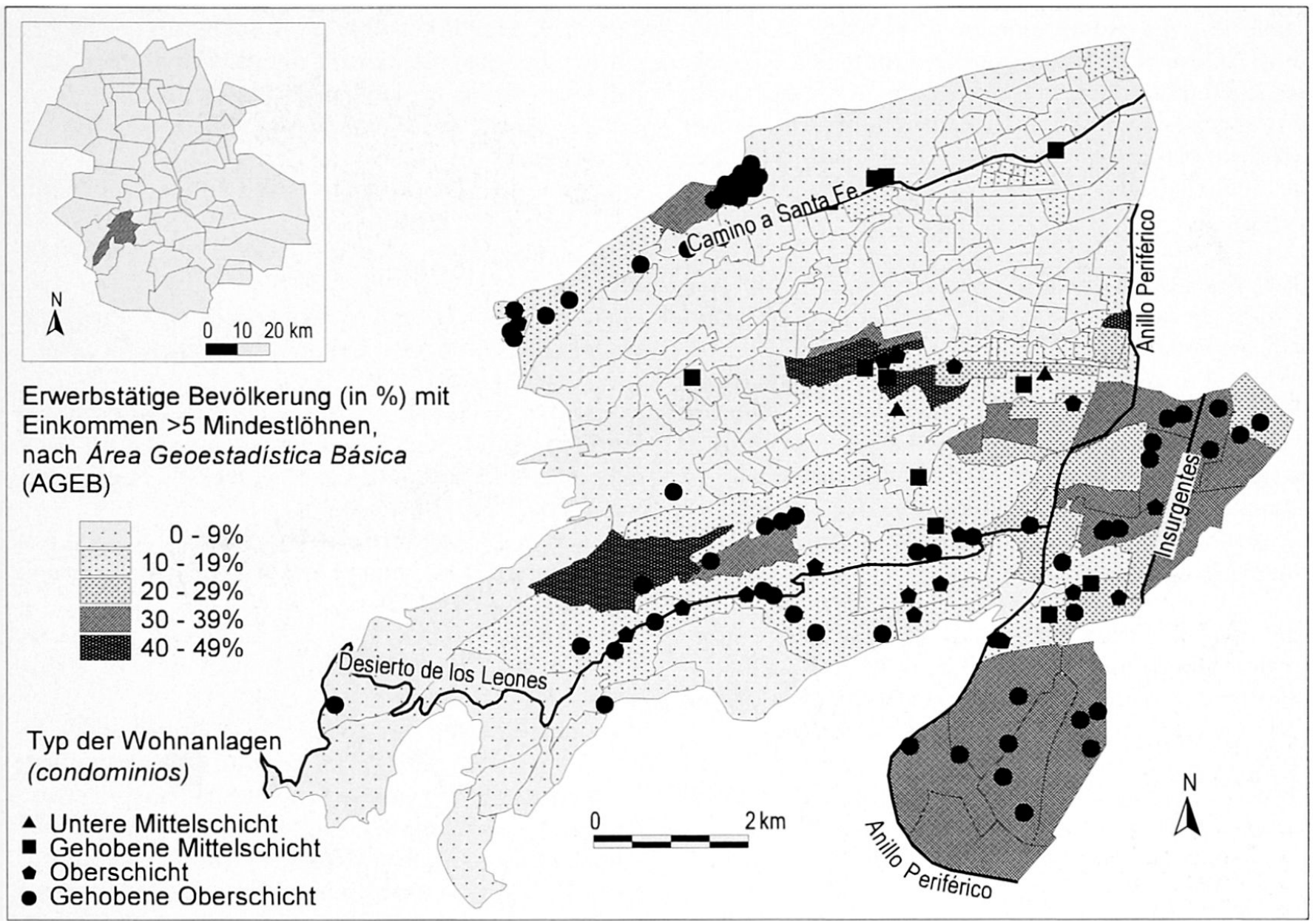

Abb. 6: Anteil der Besserverdienenden in der delegación Álvaro Obregón und Verteilung der von 1989-2001 neu gebauten condominios mit Sicherheitseinrichtungen.

Relative distribution of higher income residents and distribution of secured "condominios» built 1989-2001 in the «delegación» Álvaro Obregón.

Pourcentage des habitants à revenu supérieur dans la "delegación» Álvaro Obregón et répartition des "condominios» nouvellement construits, dotés d'équipements de sécurité (1989-2001).

Kartengrundlage: Instituto Nacional de Estadística, Geografía E Informatica 1992 und Softec S.C. 1989-2001; Kartographie: S. KANITSCHEIDER

zirke aber genauer, stellt sich heraus, dass sich innerhalb dieser recht groben West-Ost-Teilung in einem größerem Maßstab durchaus eine Heterogenität sozialer Art zeigt. Diese Unterschiede, die sich auch in den Grundstückspreisen und der Verfügbarkeit von Baugrund ausdrücken, scheinen eine Nische für die Ansiedlung von condominios cerrados der Oberschicht darzustellen. Die räumlich gesehen gute Lage ausnützend begegnen die Immobilienfirmen den «Nachteilen» der sozial niedriger gestellten Nachbarschaft durch hohe Mauern und starke Sicherheitsvorkehrungen. In diesem Sinne ist es durchaus berechtigt, von einer kleinräumigeren Segregation zu sprechen, wenn auch mit den genannten Einschränkungen.

\section{Schlussfolgerungen}

Während die Bevölkerung diese relativ neue Wohnform meist schon als selbstverständlich hinnimmt, scheinen barrios cerrados für die öffentliche Verwaltung nur dann zu existieren, wenn es Klagen über das Absperren von Straßen gibt. Aus der Perspektive der Stadtplanung und -entwicklung spielen die geschlossenen Wohnviertel nur in der akademischen Diskussion eine Rolle. Dabei handelt es sich, wie die Untersuchung zeigt, keinesfalls um ein reines Oberschichtphänomen, denn auch Bürger der mittleren und zu einem kleinen Teil sogar der unteren Einkommensklassen siedeln in barrios cerrados. Das zahlenmäßig starke Wachstum in den letzten Jahren deutet an, dass es sich bald nicht 
mehr um eine Randerscheinung handeln wird, sondern ein zu berücksichtigender Anteil der Einwohner, noch mehr allerdings der Fläche in Mexiko-Stadt von abgesperrten Wohnsiedlungen unterschiedlicher Größe betroffen sein wird. Spätestens dann wird auch die Stadtregierung sich an der Diskussion beteiligen müssen.

Obwohl die traditionellen Segregationsmuster durch die barrios cerrados im kleinräumigen Bereich eine deutliche Abänderung erfahren, ist ein Ersetzen der klassischen Armen- und Reichenviertel mittelfristig nicht abzusehen. Denn die durch diese Wohnform ermöglichte stärkere Vermischung sozialer Schichten ist begrenzt, da sich die «Insel» trotz aller Abschottungsmaßnahmen räumlich nicht von der Umgebung trennt, sondern die naturräumliche Lage und Umweltqualität mit den angrenzenden Gebieten teilt.

Auch diese Untersuchung lässt viele Fragen noch unbeantwortet und kann nicht mehr sein als ein Mosaiksteinchen im Gesamtbild, das die bereits durchgeführten und zukünftigen Untersuchungen über barrios cerrados in Mexiko und ganz Lateinamerika zeichnen werden, um letztlich lokale Besonderheiten von allgemeinen Trends trennen zu können.

\section{Dank}

Diese Arbeit wurde finanziert durch den Fonds zur Förderung wissenschaftlicher Forschung (Nummer P 14883).

\section{Literatur}

Beltrán, I. \& P. Piccato (2000): Crimen en el siglo XX: Fragmento de análisis sobre la evidencia cuantitativa. - Instituto de Investigaciones Jurídicas, Universidad Nacional Autónoma de México, Ciudad de México (unveröffentlichtes Manuskript).

Borsdorf, A. (1998): Vom Casco Colonial zum Barrio Amurallado: Wohnformen in lateinamerikanischen Städten. - In: KALLER-Dietrich, M. (Hrsg.): Recht auf Entwicklung? Atención! Jahrbuch des Österreichischen Lateinamerika-Instituts 1.- Frankfurt a.M.: 81-105.

Borsdorf, A. (2000): Condominios von Santiago de Chile als Beispiele sozialräumlicher Segregationstendenzen von Ober- und Mittelschicht in lateinamerikanischen Städten. - Peripherie 80, 25-40.

Cabrales Barajas, L.F. \& E. Canosa Zamora (2001): Segregación residencial y fragmentación urbana: los fraccionamientos cerrados en Guadalajara. - Espiral, Estudios sobre Estado y Sociedad Guadalajara 7: 223-253.

Consejo Nacional de Población (2000): Escenarios demográficos y urbanos de la Zona Metropolitana de la Ciudad e México, 1990-2010. - Síntesis, México D.F.
COY, M. \& M. Pöhler (2001): Wohnghettos der Privilegierten. Die Fragmentierung der brasilianischen Stadt. Fallbeispiele aus Rio de Janeiro und São Paulo. - Matices. Zeitschrift zu Lateinamerika, Spanien und Portugal 8 (29): 8-12.

Gobierno del Distrito Federal (1999): Reglamento de Tránsito del Distrito Federal, Art. 103, IIIj. - In: Gaceta Oficial del Distrito Federal 152, Ciudad de México.

Gobierno del Distrito Federal (2001): Programa General de Desarrollo Urbano del Distrito Federal. Ciudad de México.

Instituto Nacional de Estadística, Geografía e Informatica (1992): Sistema para la consulta de la información censal. XI Censo General de Población y Vivienda, 1990. - México.

JANOSCHKA, M. (2000): Reich und arm in Buenos Aires. Barrios privados als neue Form der Suburbanisierung. - Praxis Geographie 30 (12): 60-62.

MEYER, K. \& J. BÄHR (2001): Condominios in Greater Santiago de Chile and their Impact on the Urban Structure. - Die Erde 132 (3): 293-321.

PöHLER, M. (1999):Zwischen Luxus-Ghettos und Favelas. Stadterweiterungsprozesse und sozialräumliche Segregation in Rio de Janeiro: Das Fallbeispiel Barra da Tijuca. - = Kleinere Arbeiten aus dem Geographischen Institut der Universität Tübingen 21, Tübingen. SAFA, P. (1999): Construir mundos, levantar muros y preservar patrimonios: ¿una alternativa de vida en las grandes ciudades? Condominios y fraccionamientos cerrados en la ciudad de México. - Centro de Investigaciones y Estudios Superiores en Antropología Social (CIESAS)-Occidente, Guadalajara (unveröffentlichtes Manuskript).

Softec S.C. (Hrsg.) (1989-2001): Dinámica del mercado inmobiliario habitacional (DIME). - Ciudad de México.

SofTec S.C. (Hrsg.) (2001): Dinámica del mercado inmobiliario. Perspectiva Inmobiliaria 1. - Ciudad de México.

SuÁREZ, F. (1997): Nuevas tendencias residenciales en la Ciudad de Buenos Aires. Carta Económica Regional 52. - Instituto de Estudios Económicos y Regionales, Universidad de Guadalajara.

\section{Zusammenfassung: Condominios und fracciona- mientos cerrados in Mexiko-Stadt - Sozialräumliche Segregation am Beispiel abgesperrter Wohnviertel} Die vorliegende Arbeit dokumentiert eine Fallstudie zur sozialräumlichen Segregation am Beispiel des Wohnungsmarktes in der Stadt Mexiko. Zentraler Punkt dieser empirischen Untersuchung ist das Phänomen der gated communities (in Mexiko condominios oder fraccionamientos cerrados).

Die Analyse der sozialen Dimension dieser verhältnis- 
mäßig jungen Wohnform in der 18-Millionen-Metropole Mexiko verdeutlicht eine Verbreitung der geschlossenen Wohnsiedlungen (barrios cerrados) in den verschiedenen Gesellschaftsschichten und deren unterschiedliche Motivation für diese freiwillige Segregation. Die Untersuchung der räumlichen Verbreitung zeigt eine relative Gleichverteilung über das Stadtgebiet, jedoch mit Tendenz zu einer kleinräumiger werdenden Segregation. In zeitlicher Hinsicht lässt sich für die letzten Jahre eine deutlich steigende Attraktivität des Stadtzentrums feststellen, zugleich mit einer Zunahme des Wohnungsanteils an den insgesamt errichteten Wohneinheiten.

Trotz des bisher lokal und regional dürftigen Forschungsstandes weisen die Untersuchungsergebnisse darauf hin, dass mittelfristig die Bedeutung der barrios cerrados in Mexiko weiter zunehmen wird und die strukturellen und gesellschaftlichen Konsequenzen sie zum Thema der Stadtpolitik machen.

\section{Summary: Condominios and fraccionamientos cerrados in Mexico City - Gated communities as an example of socio-spatial segregation}

This paper presents a case study of socio-spatial segregation based on the housing market of Mexico City. The empirical investigation focuses on the phenomenon of gated communities (known in Mexico as condominios or fraccionamientos cerrados).

The analysis of the social dimension of this relatively modern type of housing in a metropolis of 18 million people brought two aspects to the fore: there is a clear increase in the establishment of gated communities (barrios cerrados) targeted at different social groups over the last decades. Further, the reasons given for this voluntary segregation reflect social stratification. The investigation revealed a relatively balanced spatial distribution of barrios cerrados in the urban area, the tendency however points towards increased smallscale segregation. From a temporal point of view, over the last few years there appears to be a growing interest in the city centre as a residential area. At the same time, the relation of apartments to single housing units in the barrios cerrados is increasing.

Observations made in this paper cannot be compared with external research results due to the lack of relevant research data available on regionally-specific aspects of the topic. However, the observations made here indicate that in the medium term, the importance of barrios cerrados will continue to increase, and related structural and social changes will have to be dealt with in urban policy making sooner or later.
Résumé: Les condominios et fraccionamientos cerrados à Mexico-ville - Ségrégation socio-spatiale à l'exemple de quartiers fermés

La présente étude porte sur un cas de ségrégation socio-spatiale relatif au marché résidentiel dans la capitale mexicaine. L'aspect central de cette investigation empirique est le phénomène des quartiers fermés (gated communities, au Mexique condominios ou fraccionamientos cerrados).

L'analyse de la dimension sociale de ce modèle d'habitation relativement récent dans la région métropolitaine de Mexico, forte de 18 millions d'habitants, révèle une diffusion des quartiers fermés dans les diverses couches sociales et des motivations différenciées en ce qui concerne cette ségrégation volontaire. L'observation de la dispersion spatiale témoigne d'une répartition urbaine relativement homogène, avec toutefois une tendance vers une ségrégation à échelle réduite. Au fil du temps, les dernières années permettent de constater clairement l'attractivité croissante du centre-ville. Nous relevons aussi la part résidentielle grandissante dans les nouvelles unités d'habitation.

En dépit du faible niveau de recherche locale et régionale, les résultats enregistrés montrent qu'à moyen terme l'importance des barrios cerrados à Mexico va s'accentuer et que les répercussions structurelles et sociétales sont appelées à en faire un thème central de la politique urbaine.

\section{Didaktische Hinweise}

- Wie werden geschlossene Siedlungen in den verschiedenen lateinamerikanischen Ländern bezeichnet?

- Welche Ursachen führten in Mexiko-Stadt zur Entstehung von barrios cerrados? Wie verlief die Entwicklung zeitlich und räumlich?

- Wie erklärt sich die soziale kleinräumige Segregation?

- Welches ist der aktuelle Trend in der Frage der Segregation?

Cand. rer. nat. Sigrun Kanitscheider, Serlesstrasse 29, A-6167 Neustift.

e-mail: Sigrun_Kanitscheider@gmx.de

Manuskripteingang/received/manuscrit entré le

11.9.2002

Annahme zum Druck/accepted for publication/accepté pour l'impression: 4.12 .2002 